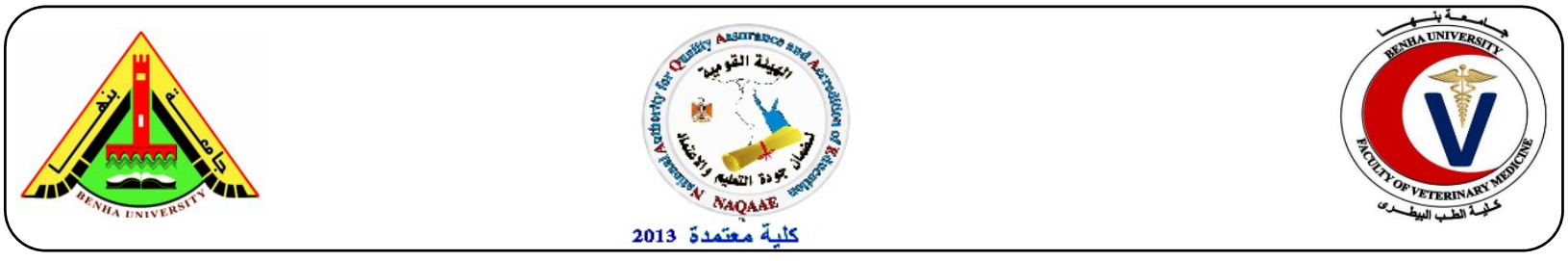

\title{
Efficacy of Inactivated Equine Influenza H3N8 Vaccine Adjuvanted with Montanide Pet Gel A
}

Eman. M. Ebied

Veterinary Serum and Vaccine Research Institute, Abbasia, Cairo, Postal No: 11381 P.O. Box 131. E-mail: Svri@idsc.gov.eg.

\section{A B S T R A C T}

This study demonstrated the use of MONTANIDE PET GEL A as polymeric adjuvant in locally prepared inactivated Equine influenza (EI) vaccine. Safety and potency of the prepared vaccine were studied by inoculation into guinea pigs and horses and evaluated using Haem agglutination inhibition (HI) test. The vaccine was proved safe and potent for horses and guinea pigs. The mean HI antibody titers in sera of guinea pigs and horses at 3 weeks post vaccination were (752.4 and 144), respectively. The antibody titers in vaccinated horses were monitored where they lasted in their sera within the protective level (80) up to 9 months post vaccination. Therefore, we recommended the use of Montanide pet gel $\mathrm{A}$ as adjuvant for horse vaccines.

Keywords: montanide pet gel, Equine Influenza, inactivated Vaccine

(http://www.bvmj.bu.edu.eg) conference issue

(BVMJ-28(2): 20-25, 2015)

\section{INTRODUCTION}

$\mathrm{E}$ quine influenza A viruses (EIV, H7N7 and H3N8 subtypes) are a leading cause of respiratory disease in horses. The H3N8 subtype of equine influenza virus remain today a serious threat to horse welfare and an economic problem for horse industry. As Equine influenza (EI) characterized by high morbidity rate which may be more than $90 \%$ (Gerber, 1970). Horses which infected by the disease and recovered without complications need supportive care and should be rested at least one week for every day of fever to allow regeneration of the mucociliary apparatus (Maanen and Cullinane, 2002) and (Bonnie and Dacvim, 2014). Vaccination is the best way for controlling the disease .The principal aim of vaccination is to reduce clinical signs of the disease, to shorten the convalescence period and reduce the likelihood of secondary infection (OIE, 2012) and (Paillot et al., 2013). Since the introduction of EI vaccines in 1960s, the majority of equine influenza vaccines commercially available contained inactivated whole virus and remain constrained by the requirement of a correct match between the vaccine and field strain of EIV. frequently using of only antigen for vaccination is not enough to stimulate immune response, it is preferable to mix the antigen with an adjuvant to boost the potency and longevity of specific immune response to antigens , (Nikolai and Julio, 2004 ), (Paillot et al, 2006) and ( Steven et al., 2008). Selecting the right adjuvant is one of the keys for success of the vaccine in the field (Parker et al., 2009). The adjuvant selected must have the best balance between safety and efficacy animal species and its sensitivity must be taken in consideration (Steven et al, 2008). Montanide GEL is an innovative polymeric adjuvant designed to improve the safety and efficacy of aqueous vaccines. It is a dispersion of highly stable gel particles of polymer (sodium polyacrylate) in water (Deville et al, 2011). The depot effect with slow release due to 
polymer adsorption properties improves the recruitment of the innate immune system. Thus in this study presented here we try to improve the strength and duration of immune response by using Montanide pet gel $\mathrm{A}$ as an adjuvant for EI vaccine.

\section{Material and Methods}

\subsection{VIRUS :}

Locally identified isolate of EIV( Magda et al.,2011) which designated as (A/equi 2/6066 NAMRU3- VSVRI/2008) egg passage number $5 \quad\left(\mathrm{EP}_{5}\right)$ with haemagglutinating ( HA ) titer $11 \log 2 / 0.05$ $\mathrm{ml}$ and infectivity titer $9 \log 10$ EID50 / 0.1 $\mathrm{ml}$ was obtained from the department of horse vaccine researches at veterinary serum and vaccine research institute (VSVRI).

\subsection{ANTISERA:}

Reference antiserum against EIV A/equi1/parague/56 (H7N7) and A/equi-2/ Miami/63 (H3N8) were obtained from National veterinary Laboratories, United States department of Agriculture, veterinary services (NVSL, USDA, VS) and used in the Identity test.

\subsection{Animals}

\subsubsection{Horses:}

Six apparently healthy seronegative horses (3- 4 years old), four of them were used to study the immune response to the prepared vaccine and two used as a control.

\subsubsection{Guinea pigs:}

Two groups of Guinea pigs 250-300 gm / weight (10 / group): the first one was used to determine the potency and safety of the prepared vaccine and the other group used as a control.

\subsection{Embryonated chicken eggs(ECE ) :}

Specific pathogen free (SPF) 9 - 11 days old ECE were used for virus propagation, egg infectivity titration and to detect the residual infective virus in the inactivated virus fluid (OIE, 2012) .

\subsection{Binary ethyleneimine (BEI):}

$0.1 \mathrm{M}$ binary ethyleneimine was dissolved in $100 \mathrm{ml}$ of $0.2 \mathrm{NaOH}$ solution (Aldrich chemical Co. LTD) and used as virus inactivator according to (Eman, 2005).

\subsection{Montanide pet gel A :}

It is an innovative, ready to disperse polymeric adjuvant was designed to improve the efficacy and safety of the vaccine and developed for companion animals. It was obtained from SEPPIC company.

\subsection{Preparation of locally inactivated egg adapted EI vaccine :}

2.7.1. Identity test: The identity of the vaccine seed EI virus (A/equi-2/ 6066 NAMRU3- VSVRI/2008) Egg passage 3 (EP3) was confirmed by haemagglutination inhibition (HI) test using reference antiserum against EIV subtype 1 and 2 (OIE, 2012).

2.7.2. Virus inactivation: according to (Eman, 2005) :vaccine virus fluid of EI subtype-2 with haemagglutinating titer (HA titer) $11 \log 2 / 0.05 \mathrm{ml}$ and infectivity titer $9 \log 10$ EID50 $/ 0.1 \mathrm{ml}$ was incubated with BEI in a final concentration $(0.003 \mathrm{M})$ at $37^{\circ} \mathrm{c}$ for 24 hours .

2.7.3. Residual Infective virus activity in ECE: It was performed according to the method described by (OIE, 2012): $0.2 \mathrm{ml}$ of the undiluted inactivated virus fluid was inoculated into the allantoic cavities of a group of fertile eggs, and incubated at 33 $35^{0} \mathrm{C}$ for 3 days. The allantoic fluid was harvested from the inoculated eggs and 0.2 $\mathrm{ml}$ of the harvested undiluted fluid was inoculated into a further group of fertile eggs. haemagglutinin activity should not be detected in the new group of eggs .

2.7.4. Sterility test: Samples from the inactivated virus fluid were cultured on different media to insure its freedom from bacterial and fungal contamination, (OIE, 2012). 
2.7.5. Vaccine formulation: Montanide pet gel A was added with a ratio $10 \%$ to the antigenic media and mixed gently according to SEPPIC Company RSS France. The vaccine was dispensed in vials , each one contain one $\mathrm{ml}$ representing the horse dose which contain $10 \log 2 \mathrm{HA}$ unites / $0.05 \mathrm{ml}$ (Soliman et al., 2008).

2.7.6.Safety of the prepared inactivated EI vaccine ( OIE, 2012 ) : Two horses, each one was inoculated $\mathrm{I} / \mathrm{M}$ with the dose of the vaccine ( $1 \mathrm{ml} /$ dose) repeated 4 weeks later. The horses are kept under observation for 10 days after the second set of injection for recording any abnormal local or systemic reaction.

\subsubsection{Potency of the prepared EI vaccine:} (OIE, 2012 ): Two groups of guinea pigs (10 guinea pigs/ group), the first group was inoculated $\mathrm{S} / \mathrm{C}$ with one horse dose $(1 \mathrm{ml})$ of the vaccine and another group left as a control at the same conditions of the experiment. Serum samples were collected 21 days post inoculation from the two groups and tested for $\mathrm{HI}$ antibody titer using HI test.

2.7.8. Immune response of horses vaccinated with the prepared EI vaccine adjuvanted with Montanide pet gel A:

Four seronegative horses, each inoculated deep I/M with the prepared EI vaccine, then they received a booster dose 6 weeks later. Two horses were left as control in the same conditions. Serum samples were collected from all horses at regular intervals, and screened for the immune response using Haemagglutination inhibition (HI) test.

\subsubsection{Haemagglutination test (HA) \&} Haemagglutination Inhibition test (HI): They were conducted according to the method described by (OIE, 2012) and used for titration of vaccine viral fluid and evaluation of the humeral immune response of immunized G. pigs and horses.

\section{RESULTS:}

Table (1): Titration of EI virus fluid
Titration of the vaccine viral fluid: The titer of the vaccine viral fluid was $11 \log 2 \mathrm{HA}$ unites $/ 0.05 \mathrm{ml}$ and its infectivity titer was 9 $\log 10$ EID50 (Table, 1). The vaccine virus fluid was completely inactivated with BEI at a final concentration $0.003 \mathrm{M}$ within 24 hours and showed no haemagglutinating activity when inoculated into ECE 9-11 days old. Freedom of the vaccine viral fluid from contamination with any biological materials was confirmed when cultured on different media. The prepared vaccine was proved to be safe when inoculated in horses. Guinea pigs serum samples of group (A) which inoculated with $1 \mathrm{ml}$ of the prepared vaccine at 3 weeks post inoculation showed mean HI antibodies titer of 752.4 while the control group (B) showed negative results (Table, 2). Table 3 and figure 1 clarified the ability of the prepared vaccine to stimulate $\mathrm{HI}$ antibodies in sera of vaccinated horses at 2 weeks post the first vaccination with a mean value (56) which rise at 3 and 4 weeks into (144) and (128) respectively, by booster at 6 week much higher level of HI antibodies were obtained (384) at 8th weeks. Antibodies reached their maximum at 4 months post vaccination (1536), then decline gradually till 9 months post vaccination but remain within protective level (80).

\section{DISCUSSION:}

Horses are sensitive species able to react strongly to vaccines compared to farm animals. Adjuvant selection during vaccine development is a key parameter driving vaccine safety and efficacy profile (Deville et al., 2011). Therefore, our study demonstrate the ability to use Montanide pet gel $\mathrm{A}$ as an adjuvant for EI vaccine. The titer of EIV (A/equi -2/6066 NAMRU3VSVRI/2008) which used for vaccine preparation was $11 \log 2 \mathrm{HA}$ unites $/ 0.05$ $\mathrm{ml}$ and infective titer was $9 \log 10$ EID $_{50} /$ $0.1 \mathrm{ml}$ as presented in table (1) where the vaccine seed virus should contain HA 


\begin{tabular}{lll}
\hline EIV (A/equi-2/6066 & HA Titer & Infectivity titer \\
NAMRU3- VSVRI/2008) & $(\log 2 / 0.05 \mathrm{ml})$ & $\left(\log 10 \mathrm{EID}_{50} / 0.1 \mathrm{ml}\right)$ \\
\cline { 2 - 3 } & 11 & 9 \\
\hline
\end{tabular}

Table (2): EI HI antibody titer in sera of guinea pigs inoculated with inactivated EI vaccine adjuvanted with montanide pet gel A using HI test

\begin{tabular}{llll}
\hline \multicolumn{4}{c}{$\begin{array}{l}\text { HI antibody titer } \\
\text { Vaccinated }\end{array}$} \\
Animal number* & $\begin{array}{c}\text { Pre- } \\
\text { inoculation }\end{array}$ & $\begin{array}{l}\text { 21 days post } \\
\text { inoculation }\end{array}$ & $\begin{array}{l}\text { Control } \\
\text { Group }\end{array}$ \\
\hline 1 & 0 & 1024 & 0 \\
2 & 0 & 1024 & 0 \\
3 & 0 & 512 & 0 \\
4 & 0 & 1024 & 0 \\
5 & 0 & 512 & 0 \\
6 & 0 & 1024 & 0 \\
7 & 0 & 512 & 0 \\
8 & 0 & 256 & 0 \\
9 & 0 & 512 & 0 \\
10 & 0 & 1024 & 0 \\
Mean & 0 & 752.4 & 0 \\
\hline r of animal in each group. Protective HI titer not less than (64) according to (OIE, 2012)
\end{tabular}

Table (3): EI HI antibody titer in sera of horses inoculated with inactivated EI vaccine adjuvanted with montanide pet gel $\mathrm{A}$ using $\mathrm{HI}$ test

\begin{tabular}{llllllll}
\hline Time of sampling & \multicolumn{5}{c}{ HI antibody titer in sera of vaccinated horses } \\
& Vaccinated group & & \multicolumn{4}{c}{ Control group } \\
& H1 & H2 & H3 & H4 & Mean & H5 & H6 \\
\hline Prevacc. & 0 & 0 & 0 & 0 & 0 & 0 & 0 \\
2 weeks & 64 & 32 & 64 & 64 & 56 & 0 & 0 \\
3 weeks & 128 & 64 & 256 & 128 & 144 & 0 & 0 \\
4 weeks & 64 & 128 & 256 & 64 & 128 & 0 & 0 \\
6 weeks (b) & 64 & 128 & 128 & 64 & 96 & 0 & 0 \\
8 weeks & 256 & 512 & 512 & 256 & 384 & 0 & 0 \\
3 months & 1024 & 1024 & 2048 & 512 & 1161 & 0 & 0 \\
4 months & 1024 & 2048 & 2048 & 1024 & 1536 & 0 & 0 \\
5 months & 1024 & 1024 & 2048 & 1024 & 1280 & 0 & 0 \\
6 months & 512 & 512 & 1024 & 1024 & 768 & 0 & 0 \\
7 months & 256 & 256 & 512 & 512 & 384 & 0 & 0 \\
8 months & 128 & 256 & 128 & 128 & 160 & 0 & 0 \\
9 months & 64 & 128 & 64 & 64 & 80 & 0 & 0 \\
10 months & 32 & 64 & 32 & 32 & 40 & 0 & 0 \\
11 month & 32 & 32 & 16 & 32 & 28 & 0 & 0 \\
12 month & 16 & 16 & 8 & 16 & 14 & 0 & 0 \\
\hline
\end{tabular}

(H): HORSE. (b): booster vaccination. Protective HI titer not less than (64) according (OIE, 2012) 
Fig (1): Seroconversion of horses inoculated with inactivated EI vaccine adjuvanted with Montanide pet gel A tested by HI test. W: weeks, M: months. B: booster dose

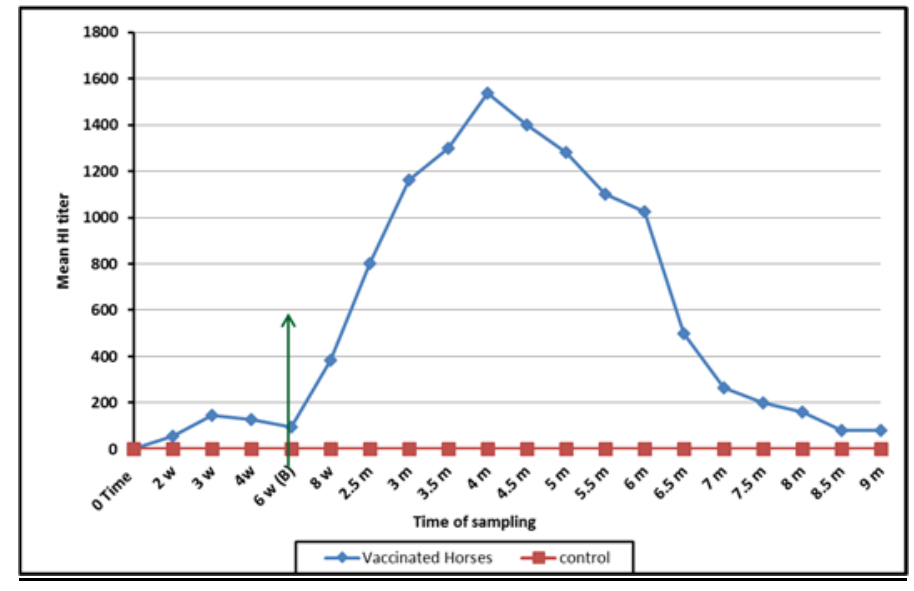

unites not less than $9 \log 2$ HA unites /0.05 $\mathrm{ml}$ and $7 \log 10 \mathrm{EID}_{50} / 0.1 \mathrm{ml}$ as recorded by (Kucera and Beckenhauer, 1977) and (Eman et al, 2009). The vaccine virus fluid was completely inactivated with BEI at a final concentration $0.003 \mathrm{M}$ within 24 hours (Eman, 2005). The inactivated vaccine virus fluid showed no haemagglutinin activity when inoculated into ECE 9 - 11 days old (OIE, 2012). The freedom of the vaccine virus fluid from contamination with biological materials was confirmed by sterility tests. No untoward local or systemic reactions were observed on horses vaccinated with the prepared vaccine confirming vaccine safety as recommended by (OIE, 2012). The potency of the prepared vaccine in guinea pigs was demonstrated by the obtained data in table (2): guinea pig serum samples of group (A) which inoculated with $1 \mathrm{ml}$ of EI vaccine adjuvanted with montanide pet gel $\mathrm{A}$ at 3 weeks post inoculation showed mean $\mathrm{HI}$ antibodies titer of 752.4. While the control group (B) showed negative results where the protective HI antibodies titer should not less than 64 according to the European pharmacopoeia (2005) and (OIE, 2012). The ability of EI vaccine to invoke Seroconversion in horses with negative prevaccination HI antibody titer was shown in Table (3), which clarify the occurrence of $\mathrm{HI}$ antibodies in sera of horses at 2 weeks post the first vaccination with mean value
(56) which rise at 3 and 4 weeks into (144) and (128) respectively. By booster at 6 weeks, much higher level of HI antibodies were obtained (384) at 8th weeks. Sera, which collected at 4 months post vaccination had the highest $\mathrm{HI}$ antibody titers (1536), and then the titer began to fall gradually in all horses till the $9^{\text {th }}$ month post immunization. The antibodies still exist in sera of horses until the $9^{\text {th }}$ month with a considerable protective titer (80) where the recorded protective $\mathrm{HI}$ antibody titer is (64) according to the European pharmacopoeia, (2005) and OIE, (2012). The results of the current study agree with the previous results obtained by European pharmacopoeia, (2005); Eman et al., (2009) and OIE, (2012) who stated that EI vaccine induce high level of antibodies when add a suitable adjuvant to the antigenic media. Also Parker et al., (2009) who recorded that Montanide gel can be safely used associated with various antigenic media and animal species with protective immune response and safety profile more than aluminum salts, and (Deville et al., 2011) who decided that Montanide pet gel A based vaccines in horses showed no impact on the animal and induced antibodies with a level higher than aluminum based vaccines.

From the obtained results, it could be concluded that MONTANIDE PET GE L A could be used to enhance the 
immune response of horses to EI vaccine with a safety profile.

\section{REFRENCES:}

Bonni, R.R. and Dacvim DVM. 2014. Last full review /revision journal 2014. The Merk veterinary Manual

Deville, S.; Bertrand, F.; Carneux, E.; Cauchard, S.; Cauchard, J. and Dupuis, L. 2011. "Adjuvant formulation for companion animal vaccines" Procedia in vaccinology 4:104-112. $4^{\text {th }}$ vaccine and ISV Annual global congress.

Eman 2005."Trials for preparation of killed adjuvanted vaccine for Equine influenza virus" $\mathrm{PhD}$. thesis. faculty of veterinary medicine, Cairo university.

Eman, M. Ebied ; Nehal, S. Saleh ; Nashwa K. Madkour ;Elkabbany M.M.A. and Soliman I.M.A. (2009) : " Trials for preparation of monovalent inactivated Equine influenza vaccine" . Third 1 Sci. conf., Banha \& Ras Sudr, EGYPT.

European pharmacopoeia 2005. Equine influenza vaccine (inactivated). 01/ 2005:0249, pp. 755 - 757.

Gerber, H. 1970. Sequelae and epidemiology of equine influenza. in equine infectious diseases , $112^{\text {nd }}$ Int Conf. on Equine inf. Di., Paris 1969 Karger P. 69

Kucera, C.j. and Beckenhauer, W.H. 1977. "Studies on the antigenicity of an inactivated aluminium hydroxide adjuvant equine influenza vaccine ." CAN. J. Comp. Med., 44(3):326- 331.

Maanen, C.V. and Cullinane, A. 2002. "Equine influenza virus infection: an update " Veterinary Quarerly 24(2):79 $-94$

Magda A. Kalad ; Eman M. Ebied ; Nashwa K. Madkour; Safaa A. Warda; Nehal S.
Saleh; Elkabbany, M. M.A.; Soliman, I. M.; Magdi, D. Saad; Deidrashuck. Lee; Mary, A. Younan; Emad, M. Eassal; Jeffrey A. Tjaden 2011. "Characterization of Equine influenza A virus H3N8 isolate in Egypt 2008". Ippologia, Anno 22(3):35-44.

Nikolai Petrovsky and Julio Cesar Aguilar 2004. "Vaccine adjuvants: Current state and further trends". Immunology and cell biology.

OIE May, 2012. Equine influenza Chapter 2.5.7. $82,488-496$

Paillot, R.; Hannant, D.; Kydd, J.H.; and Daly, J.M. 2006. "Vaccination against equine influenza: Quid novi vaccine ". Animal health Trustcenter for preventive medicine. 24:4047-4061

Paillot, R.; Prowse, L.; Montesso, F.; Huang, C.M. ; Bames, H. ; and Escala, J. 2013. " Whole inactivated equine influenza vaccine: Efficacy against a representative clad 2 equine influenza virus, IFNgamma synthesis and duration of humoral immunity" . Vet. microbiology 162(2-4): 396-407

Parker, R.; Deville, S.; Dupuis, L.; Bertrand, F. and Aucouturier, J. 2009. "Adjuvant formulation for veterinary vaccines montanide ${ }^{\mathrm{TM}}$ safety profile" Procedia in vaccinology 1(1):140-147

Soliman, I.M.A.; M.M.A. El-Kabbany; Magda A. Kalad; Nashwa K. Madkour; EmanM. Ebied; Nehal S. Saleh; Safaa A. Warda and H.I. Smaha, 2008. "Recent isolation and identification of equine influenza (EI) in Egypt, 2008." Ninth Scientific Veterinary Medical Conference, pp. 747-756.

Steven, G. Reed, Sylvie Bertholet, Rhea N. Coler and Martin Friede 2008. " New horizons in adjuvants for vaccine development " Trend immunology 30: $23-32$. 